\title{
Impaired Set-Shifting Ability in Patients with Eating Disorders, Which Is Not Moderated by Their Catechol-O-Methyltransferase Val158Met Genotype
}

\author{
Youl-Ri Kim ${ }^{\bowtie}$, Ji Eun Kim and Mi Hyun Kim \\ Department of Neuropsychiatry, Seoul Paik Hospital, Inje University College of Medicine, Seoul, Korea
}

The aim of this study was to examine the set-shifting ability in women with both anorexia nervosa (AN) and bulimia nervosa (BN) and to investigate whether it is contributed by the catechol-O-methyltransferase (COMT) Val158Met genotype. A total of 102 Korean participants-40 women with lifetime AN, 28 women with lifetime BN, and 34 healthy women of comparable age and intelligence quotientwere examined. A neuropsychological battery of tests was applied and blood samples were obtained for COMT Val158Met genotyping. Set-shifting impairments Trail Making Test (TMT, Part B) were found in patients with AN and BN, respectively. Furthermore, the eating disorders were also linked to deficits in attentional mechanisms (TMT, Part A) and motor skills (Finger Tapping Test). Finally, set-shifting and its link to eating disorders were not moderated by COMT Val158Met genotype.

Psychiatry Investig 2010;7:298-301

Key Words Eating disorders, Set-shifting ability, Anorexia nervosa, Bulimia nervosa, Catechol-O-methyltransferase.

\section{INTRODUCTION}

Eating disorders aggregate in family, ${ }^{1}$ and the variance in heredity lies between $33 \%$ and $84 \%$ in twin studies. ${ }^{2}$ Using endophenotypic markers may have great potential for finding the candidate genes, as they are generally less complex than their associated phenotype and, thus, may be more readily linked to a specific genetic locus. ${ }^{3}$ It has been suggested that the cognitive behavioral inflexibility observed in patients with eating disorders is linked to their set-shifting problem. ${ }^{4}$ The setshifting ability is considered as a candidate endophenotype of anorexia nervosa (AN), ${ }^{5}$ because pairs of sisters one of whom has AN as well as women with AN, tend to have delayed setshifting. ${ }^{6}$ Whereas there is accumulating evidence for impaired set-shifting ability in AN, much remains unknown with respect to bulimia nervosa $(\mathrm{BN}))^{7}$ The catechol-O-methyltransferase $(C O M T)$ gene is one of the candidate genes for im-

Received: May 10, 2010 Revised: August 6, 2010

Accepted: September 3, 2010 Available online: November 17, 2010

$\triangle$ Correspondence: Youl-Ri Kim, MD, PhD

Department of Neuropsychiatry, Seoul Paik Hospital, Inje University College of Medicine, 85 Jeo-dong 2-ga, Jung-gu, Seoul 100-032, Korea

Tel: +82-2-2270-0063, Fax: +82-2-2270-0344, E-mail: youlri.kim@paik.ac.kr

(a) This is an Open Access article distributed under the terms of the Creative Commons Attribution Non-Commercial License (http://creativecommons.org/licenses/bync/3.0) which permits unrestricted non-commercial use, distribution, and reproduction in any medium, provided the original work is properly cited. paired cognitive flexibility. The COMT Val158Met functional polymorphism appears to selectively impact prefrontal dopamine regulation and this gene has been shown to predict performance on prefrontal executive cognition and working memory tasks in healthy individuals. ${ }^{8}$

In the present study, we hypothesized that individuals with BN as well as AN would show impaired set-shifting ability. An extensive review of the previous literature guided our choice of the Trail Making Test (TMT) $)^{9}$ as the measure to use for the set-shifting ability, as it is the most commonly employed setshifting measure with the acceptable and consistent effect size. ${ }^{7}$ We also attempted to investigate whether their set-shifting ability is affected by the COMT Val158Met genotype. To the best of our knowledge, this is the first study to examine the possible association between endophenotypes and liable genes in eating disorders.

\section{METHODS}

A total of 102 women, comprising 40 patients with AN and 28 patients with $\mathrm{BN}$, as well as 34 healthy controls, participated in this study. The diagnosis of lifetime AN or BN was made using the Eating Disorders Examination Questionnaire Version (EDE-Q) ${ }^{10}$ on the basis of the hierarchical model of diagnosis used in a genetic study. ${ }^{11}$ Neuropsychological tests were ap- 
plied to all participants and their blood samples were collected for the COMT Val158Met genotyping. The exclusion criteria for all participants were a history of neurologic illness, brain injury, substance-abuse disorders, or psychosis in the past. Only the use of selective serotonin reuptake inhibitors (SSRIs) was permitted within 2 weeks before the neuropsychological test. Nineteen (48\%) of AN and fifteen (54\%) of BN were taking SSRIs at the time of the study. The Korean version of the Wechsler Adult Intelligent Scale was administered to match the groups in terms of their general intelligence. ${ }^{12}$ The patients underwent an interview for measuring their symptoms of eating disorders by using the Korean version of the 12th edition of the EDE. ${ }^{13}$ Their comorbid clinical symptoms were measured by the Korean versions of the Beck Depression Inventory (BDI), ${ }^{14}$ the Spielberger State and Trait Anxiety Inventory, ${ }^{15}$ and the Maudsley Obsessive Compulsive Inventory ${ }^{16}$ respectively. The participants completed various computerized neuropsychological tests spanning the cognitive domains of the TMT Part B for set-shifting ability, the Visual Span Test (VST; Corsi block tapping test ${ }^{17}$ for visual/working memory, the TMT Part $\mathrm{A}^{9}$ for attention, and the Finger Tapping Test $(\mathrm{FTT})^{18}$ for motor function. A meta-analysis revealed no evidence of publication bias for the TMT Part $\mathrm{B}^{7}$ and, therefore, the uncorrected data is presented in this study. According to Korean normative data, the test/retest reliability coefficients of the computerized tests were acceptable. ${ }^{19,20}$ Deoxyribonucleic acid (DNA) was extracted from blood leukocytes by using a commercial DNA extraction kit (ABI, Foster City, CA, USA). Genotyping of the COMT Val158Met variant was carried out with a polymerase chain reaction (PCR)-based restriction fragment length polymorphism analysis by using the primers 5'-CTCATCACCA TCGAGATCAA-3' and 5'-CCAGGTCTGAAACGGGTCA-3' The PCR products digested with NlaIII were electrophoresed to distinguish between the Val and Met alleles. This study was approved by the Institutional Review Board of Seoul Paik Hospital in Seoul, Korea. Written informed consent was obtained from all of the participants.

Group differences in the neuropsychological tests were tested in a one-way analysis of covariance with the years of education included as a covariate, since the AN group had a shorter educational years than the other groups (AN: 12.70 \pm 3.38 years, $\mathrm{BN}$ : $13.57 \pm 2.20$ years, controls: $14.65 \pm 1.87$ years; $\mathrm{F}=$ 4.99, $\mathrm{df}=2 / 99, \mathrm{p}=0.009$ ). For the set-shifting ability, a multivariate analysis of variance (MANOVA) in the groups was computed by integrating the two scores of the TMT Part B (total errors, time taken). The MANCOVAs were repeated using the general psychiatric measures as covariates to investigate the contribution of general psychiatric symptoms on the performance on the neuropsychological tests. We carried out the subsidiary analysis to test the effect of medication on the per- formance of the neuropsychological tests with a comparison of the patients in the medicated subgroup with those in the non-medicated subgroup. For effect of the COMT genotype on the set-shifting ability, a two way MANOVA for integrating the time taken and total errors of the TMT Part B was computed with the two factors, genotype and group. Two-tailed tests were used and a p-value $<0.05$ was considered to be indicative of statistical significance.

\section{RESULTS}

The clinical characteristics, the frequency of genotypes for the COMT Val158Met, and the performance on the neuropsychological tests of the participants are shown in Table 1. Both the AN and BN groups showed significantly poorer performance on the TMT Part A and FTT than the controls, but not on the VST. The MANOVA showed a significant effect of diagnosis on the set-shifting ability with Pillai's $\mathrm{F}=4.48, \mathrm{df}=4 / 196, \mathrm{p}=$ 0.002 on the TMT Part B. Depression was slightly associated with the performance on the set-shifting task $(\mathrm{F}=3.38, \mathrm{df}=2 / 93$, $\mathrm{p}=0.038$ ), but the effect of the group were also significant for the score of $\mathrm{BDI}$ as a covariate $(\mathrm{F}=3.36, \mathrm{df}=4 / 188, \mathrm{p}=0.011)$. There was no evidence that anxiety, obsessive-compulsiveness, or symptoms of eating disorders was associated with the setshifting ability. In a subsidiary analysis, the results revealed no significant group differences across all neuropsychological tasks in comparisons of the women with eating disorders who were or were not currently receiving antidepressants. We could not find any main effect of the COMT Val158 Met genotype (F= $0.54, \mathrm{df}=4 / 184, \mathrm{p}=0.703)$, group $(\mathrm{F}=2.41, \mathrm{df}=4 / 184, \mathrm{p}=0.051)$, or any interaction effects between the genotype and group $(\mathrm{F}=$ $0.50, \mathrm{df}=8 / 184, \mathrm{p}=0.858)$ on the performance of the set-shifting ability.

\section{DISCUSSION}

In this study, we reported set-shifting impairments were found in patients with $\mathrm{AN}$ and $\mathrm{BN}$, respectively. Furthermore, the eating disorders were also linked to deficits in attentional mechanisms and motor skills. This study furthers our understanding of the possibility of set-shifting deficit being used as an endophenotype of BN as well as AN.

These neuropsychological data are consistent with previous evidence of there being an impairment in attention and motor skill in underweight and recovered AN and BN patients, but no differences in the working memory or speed of memory. ${ }^{21}$ Although the performance on the set-shifting task was related to depression, the set-shifting deficit still remained after controlling for the effect of depression, indicating the deficit is a feature independent of the comorbidities. 
Table 1. The clinical characteristics, genotypes, and performance of neuropsychological tasks of the participants

\begin{tabular}{|c|c|c|c|c|c|}
\hline \multirow{2}{*}{ Variables } & \multirow{2}{*}{$\begin{array}{c}\mathrm{AN} \\
(\mathrm{A} ; \mathrm{N}=40)\end{array}$} & \multirow{2}{*}{$\begin{array}{c}\mathrm{BN} \\
(\mathrm{B} ; \mathrm{N}=28)\end{array}$} & \multirow{2}{*}{$\begin{array}{c}\mathrm{HC} \\
(\mathrm{C} ; \mathrm{N}=34)\end{array}$} & \multicolumn{2}{|c|}{ Analysis } \\
\hline & & & & Statistics (df) & $\mathrm{p}$ \\
\hline \multicolumn{6}{|l|}{ Clinical characteristics } \\
\hline Age (years) & $22.78 \pm 7.23$ & $23.00 \pm 3.21$ & $22.65 \pm 3.57$ & $F(2,99)=0.04$ & 0.966 \\
\hline Age of onset (years) & $18.55 \pm 4.59$ & $20.07 \pm 2.99$ & N/A & $t(65)=1.52$ & 0.133 \\
\hline Current BMI $\left(\mathrm{kg} / \mathrm{m}^{2}\right)$ & $16.67 \pm 2.60$ & $20.66 \pm 2.65$ & $21.52 \pm 1.68$ & $F(2,99)=44.96$ & $<0.001$ \\
\hline Lowest ever BMI $\left(\mathrm{kg} / \mathrm{m}^{2}\right)$ & $14.60 \pm 1.51$ & $8.12 \pm 2.19$ & $20.19 \pm 1.52$ & $F(2,99)=99.25$ & $<0.001$ \\
\hline WAIS-IQ & $108.50 \pm 9.61$ & $108.75 \pm 11.85$ & $107.53 \pm 9.07$ & $F(2,99)=1.33$ & 0.876 \\
\hline BDI & $19.87 \pm 13.60$ & $18.64 \pm 11.91$ & $5.82 \pm 5.95$ & $\mathrm{~F}(2,99)=16.84$ & $<0.001$ \\
\hline STAI & $106.58 \pm 25.65$ & $110.52 \pm 25.20$ & $81.76 \pm 22.74$ & $\mathrm{~F}(2,99)=12.73$ & $<0.001$ \\
\hline K-MOCI & $39.40 \pm 6.09$ & $38.24 \pm 5.16$ & $35.88 \pm 4.11$ & $F(2,99)=4.08$ & 0.020 \\
\hline EDE, global & $61.26 \pm 21.99$ & $78.21 \pm 21.99$ & N/A & $\mathrm{t}(65)=3.11$ & 0.003 \\
\hline \multicolumn{6}{|l|}{ COMT Val158Met genotype } \\
\hline $\mathrm{Val} / \mathrm{Val}$ & $22(55 \%)$ & $14(50 \%)$ & $16(47 \%)$ & \multirow{3}{*}{\multicolumn{2}{|c|}{$\begin{array}{l}\text { A vs } C: \chi^{2}(2)=1.27, p=0.53 \\
B \text { vs } C: \chi^{2}(2)=0.69, p=0.71\end{array}$}} \\
\hline Val/Met & $14(35 \%)$ & $11(39 \%)$ & $16(47 \%)$ & & \\
\hline Met/Met & $4(10 \%)$ & $3(11 \%)$ & $2(6 \%)$ & & \\
\hline \multicolumn{6}{|l|}{ Set-shifting ability } \\
\hline \multicolumn{6}{|l|}{ Trail Making Test, Part B } \\
\hline Time & $39.28 \pm 12.22$ & $49.54 \pm 33.24^{* *}$ & $31.35 \pm 7.99$ & 6.45 & 0.002 \\
\hline Errors & $5.86 \pm 8.79^{* *}$ & $6.43 \pm 10.05^{* *}$ & $0.82 \pm 1.49$ & 5.23 & 0.007 \\
\hline \multicolumn{6}{|l|}{ Attention } \\
\hline \multicolumn{6}{|l|}{ Trail Making Test, Part A } \\
\hline Time & $25.95 \pm 8.88^{* *}$ & $24.46 \pm 7.21^{* *}$ & $19.41 \pm 4.46$ & 7.25 & 0.001 \\
\hline Errors & $4.33 \pm 7.26^{*}$ & $4.64 \pm 8.16^{*}$ & $0.82 \pm 1.06$ & 3.47 & 0.035 \\
\hline \multicolumn{6}{|l|}{ Visual/Working memory } \\
\hline \multicolumn{6}{|l|}{ Visual Span Test } \\
\hline Forward & $6.33 \pm 0.96$ & $6.76 \pm 1.17$ & $6.29 \pm 0.91$ & 1.97 & 0.144 \\
\hline Backward & $5.43 \pm 0.89$ & $5.55 \pm 0.94$ & $5.72 \pm 0.66$ & 1.62 & 0.204 \\
\hline \multicolumn{6}{|l|}{ Motor skill } \\
\hline \multicolumn{6}{|l|}{ Finger Tapping Test } \\
\hline Right, taps & $53.91 \pm 8.40^{* *}$ & $56.30 \pm 5.91^{*}$ & $61.50 \pm 6.01$ & 7.45 & 0.001 \\
\hline Left, taps & $48.61 \pm 6.41^{*}$ & $48.18 \pm 9.63^{* *}$ & $54.83 \pm 4.82$ & 7.00 & 0.001 \\
\hline
\end{tabular}

Data are shown as mean \pm SD or number (percentage) as appropriate. The differences in the genotype frequencies between the patients (AN, $\mathrm{BN}$ ) and the healthy controls were evaluated using a $\chi^{2}$ test. Analysis by ANCOVA for neuropsychological tasks as covariate of years of education. ${ }^{*} \mathrm{p}<0.05,{ }^{* *} \mathrm{p}<0.01$ compared on cognitive performance as contrasted with controls. N/A: no normative data given, AN: anorexia nervosa, BN: bulimia nervosa, HC: healthy controls, BMI: Body Mass Index, K-WAIS: Korean-Wechsler Adult Intelligent Scale, BDI: Beck Depression Inventory, STAI: State and Trait Anxiety Inventory, K-MOCI: Korean version of Maudsley Obsessive and Compulsive Inventory, EDE: Eating Disorders Examination Interview

In our study, the COMT Val158Met genotype was not involved in the impaired set-shifting ability in both AN and BN. Despite this finding, there is no doubt that genetic variants are associated with the endophenotype (viz. set-shifting deficit). It may be more useful to use of the endophenotypic marker for uncovering genetic commonalities across psychiatric disorders than for a single disorder (viz. eating disorder) as setshifting appears to broadly increase the risk of many forms of psychiatric illness. ${ }^{22-24}$ The limitations of the study were to use of the TMT Part B solely as a measure of the set-shifting ability, and the relatively small sample size, which may reduce its power to detect an association. Furthermore, the multiple testings to examine links between the set-shifting deficit and possible genetic markers might increase the probability of type 1 errors.

In conclusion, our findings suggest that impaired set-shift- 
ing ability is a common feature for both $\mathrm{AN}$ and $\mathrm{BN}$ patients and its link to eating disorders were not moderated by the $\mathrm{CO}$ MT Val158Met genotype.

\section{Acknowledgments}

This work was supported by a Korea Research Foundation Grant funded by the Korean Government (MOEHRD) (KRF-2006-331-E00203).

\section{REFERENCES}

1. Strober M, Freeman R, Lampert C, Diamond J, Kaye W. Controlled family study of anorexia nervosa and bulimia nervosa: evidence of shared liability and transmission of partial syndromes. Am J Psychiatry 2000; 157:393-401.

2. Bulik CM. Exploring the gene-environment nexus in eating disorders. J Psychiatry Neurosci 2005;30:335-339.

3. Glahn DC, Bearden CE, Niendam TA, Escamilla MA. The feasibility of neuropsychological endophenotypes in the search for genes associated with bipolar affective disorder. Bipolar Disord 2004;6:171-182.

4. Tchanturia K, Anderluh MB, Morris RG, Rabe-Hesketh S, Collier DA, Sanchez P, et al. Cognitive flexibility in anorexia nervosa and bulimia nervosa. J Int Neuropsychol Soc 2004;10:513-520.

5. Bulik CM, Hebebrand J, Keski-Rahkonen A, Klump KL, ReichbornKjennerud T, Mazzeo SE, et al. Genetic epidemiology, endophenotypes, and eating disorder classification. Int J Eat Disord 2007;40:S52-S60.

6. Holliday J, Tchanturia K, Landau S, Collier D, Treasure J. Is impaired setshifting an endophenotype of anorexia nervosa? Am J Psychiatry 2005; 162:2269-2275.

7. Roberts ME, Tchanturia K, Stahl D, Southgate L, Treasure J. A systematic review and meta-analysis of set-shifting ability in eating disorders. Psychol Med 2007;37:1075-1084.

8. Barnett JH, Jones PB, Robbins TW, Müller U. Effects of the catecholO-methyltransferase Val158Met polymorphism on executive function: a meta-analysis of the Wisconsin Card Sort Test in schizophrenia and healthy controls. Mol Psychiatry 2007;12:502-509.

9. Reitan RM. Validity of the trail making test as indicator of organic brain damage. Percept Mot Skills 1958;8:271-276.

10. Fairburn CG, Beglin SJ. Assessment of eating disorders-interview or selfreport questionnaire? Int J Eat Disord 1994;16:363-370.

11. Kaye WH, Lilenfeld LR, Berrettini WH, Strober M, Devlin B, Klump
KL, et al. A search for susceptibility loci for anorexia nervosa: methods and sample description. Biol Psychiatry 2000;47:794-803.

12. Yum TH, Park YS, Oh KJ, Kim JG, Lee HY. The manual of KoreanWechsler adult intelligence scale. Seoul: Korean Guidance Press; 1992.

13. Heo SY, Rhee MK, Choi YM, Sohn CH, Lee HK, Lee YH. Reliability and factor analysis of the Korean version of eating disorder examination. J Korean Soc Study Obes 2004;13:42-52.

14. Han HM, Yeom TH, Shin YW. The validity and reliability of Beck depression inventory Korean version. J Korean Neuropsychiatr Assoc 1986;25:487-500.

15. Kim JT, Shin DK. A study based on the standardization of the state and trait anxiety inventory for Korea. New Med J 1978;21:69-75.

16. Min BB, Won HT. Reliability and validity of the Korean translations of Maudsley obsessional-compulsive inventory and Padua inventory. Korean J Clin Psychol 1999;18:163-182.

17. Corsi PM. Human memory and the medial temporal region of the brain: Dissertation Abstracts International, 34(02), 891B (University Microfilms No. AA105-77717). 1972.

18. Spreen O, Strauss E. A compendium of neuropsychological tests. 2nd edition. New York: Oxford University Press; 1998.

19. Ha KS, Kwon JS, Lyoo IK. Development and standardization of the computerized attention assessment for Korean adults. J Korean Neuropsychiatr Assoc 2002;41:335-346.

20. Kwon JS, Lyoo IK, Hong KS, Yeon BK, Ha KS. Development and standardization of the computerized memory assessment for Korean adults. J Korean Neuropsychiatr Assoc 2002;41:347-362.

21. Bosanac P, Kurlender S, Stojanovska L, Hallam K, Norman T, McGrath C, et al. Neuropsychological study of underweight and "weight-recovered" anorexia nervosa compared with bulimia nervosa and normal controls. Int J Eat Disord 2007;40:613-621.

22. Snitz BE, Macdonald AW 3rd, Carter CS. Cognitive deficits in unaffected first degree relatives of schizophrenia patients: a meta-analytic review of putative endophenotypes. Schizophr Bull 2006;32:179-194.

23. Clark L, Sarna A, Goodwin GM. Impairment of executive function but not memory in first-degree relatives of patients with bipolar I disorder and in euthymic patients with unipolar depression. Am J Psychiatry 2005; 162:1980-1982.

24. Treasure JL. Getting beneath the phenotype of anorexia nervosa: the search for viable endophenotypes and genotypes. Can J Psychiatry 2007; 52:212-219. 